\title{
Editorial
}

\section{Sustainable Mobile Computing and Communications}

\author{
Karl Andersson, ${ }^{1}$ Eric Rondeau, ${ }^{2}$ Ah-Lian Kor, ${ }^{3}$ and Dan Johansson ${ }^{4}$ \\ ${ }^{1}$ Pervasive and Mobile Computing Laboratory, Luleå University of Technology, 93187 Skellefteå, Sweden \\ ${ }^{2}$ Université de Lorraine, BP 70239, 54506 Vandoeuvre-lès-Nancy, France \\ ${ }^{3}$ School of Computing, Creative Technologies and Engineering, Leeds Beckett University, Headingley Campus, Leeds LS6 3QS, UK \\ ${ }^{4}$ Department of Informatics, Umeå University, 90187 Umeå, Sweden
}

Correspondence should be addressed to Karl Andersson; karl.andersson@ltu.se

Received 8 August 2017; Accepted 9 August 2017; Published 2 October 2017

Copyright (C) 2017 Karl Andersson et al. This is an open access article distributed under the Creative Commons Attribution License, which permits unrestricted use, distribution, and reproduction in any medium, provided the original work is properly cited.

The International Telecommunications Union has provided vital statistics on ICT use, which evidently shows an increasing trend of universal growth in ICT uptake. According to ITU, in 2013, there are almost as many mobile-cellular subscriptions as people in the world. This is attributed to the mobile revolution, which delivers ICT applications in education, business, government, banking, health, and so on. Thus, the concept of mobile computing has revolutionized people's lives such that, nowadays, any information can be accessed and transmitted anytime and anywhere from any device. This new way of interacting with services basically has two main underlying drivers: (1) availability of innovative mobile devices (e.g., smartphones and tablets) and (2) emerging ubiquitous and affordable wireless networks. According to IndustryWeek, mobile computing is accelerating in organizations as well and mobile technology is viewed as providing the biggest boost to businesses. By 2020, the number of connected devices globally is expected to reach 80 billion and augmented reality facilitated via mobile devices will penetrate most sectors: leisure, social media, retail, restaurants, property, transport, and so on.

However, mobile computing is also a power-hungry technology. Therefore, there is a pressing need for the deployment of sustainable mobile computing and communication which focus on reduced energy consumption of its mobile ICT infrastructure and $\mathrm{CO} 2$ emissions in order to uphold the three pillars of sustainability: environmental, economic, and social. This is made possible if sustainable mobile computing platforms appropriately address the following challenges: extension of battery life, reduction of heat dissipation from components, power consumption, energy efficient wireless communications, efficient resource management, and sustainable power architecture. Some of the energy efficient techniques in sustainable mobile computing are as follows: (i) hardware: homogenous and heterogeneous multiprocessor system-on-chip, integration of multicore processors and multiple accelerometers into mobile system-on-chip, contextaware and energy aware operating systems, energy aware virtual memory (PAVM), application processor integration, and optimized scheduling in multiprocessor architectures; (ii) Wi-Fi communication: energy aware cellular data scheduling and energy-delay trade-off and dynamic voltage and frequency scaling (DVFS); (iii) sensors: accuracy and energy trade-off; (iv) applications: energy efficient computation off-loading, multithreaded applications, and green software design patterns.

The scope of this special issue is in line with recent contributions from academia and industry on the recent activities that tackle the technical challenges making mobile computing and communications more sustainable. For the current issue, we are pleased to introduce a collection of papers covering a range of topics such as frameworks for multicore mobile computing; architectures for IoT with mobile fog servers; energy efficient schemes for video streaming on mobile devices; security service chaining solutions for sustainable mobile edge computing; content downloading techniques for VANETs. 


\section{Acknowledgments}

As always, we appreciate the high quality submissions from authors and the support of the community of reviewers.

Karl Andersson

Eric Rondeau

Ah-Lian Kor

Dan Johansson 

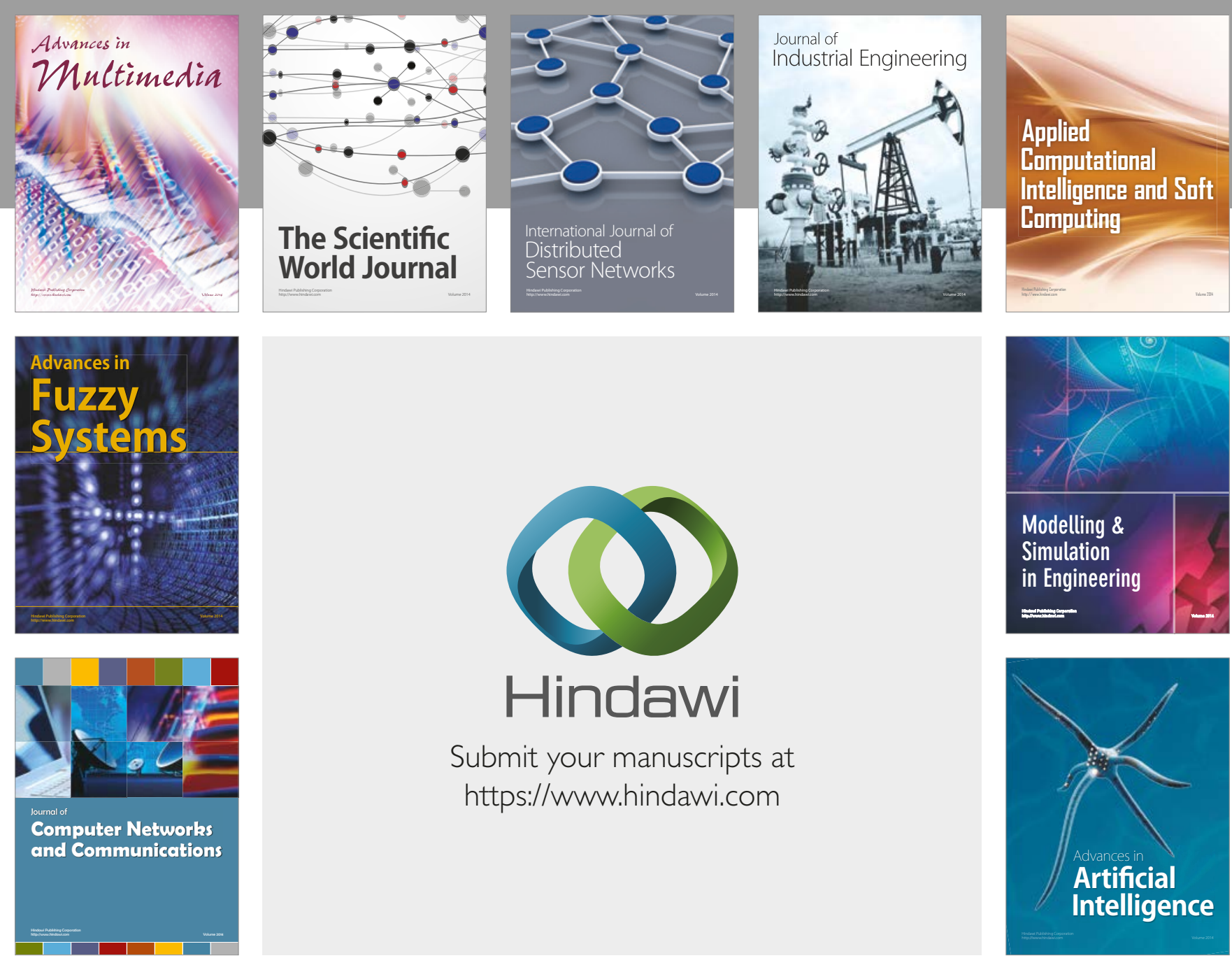

\section{Hindawi}

Submit your manuscripts at

https://www.hindawi.com
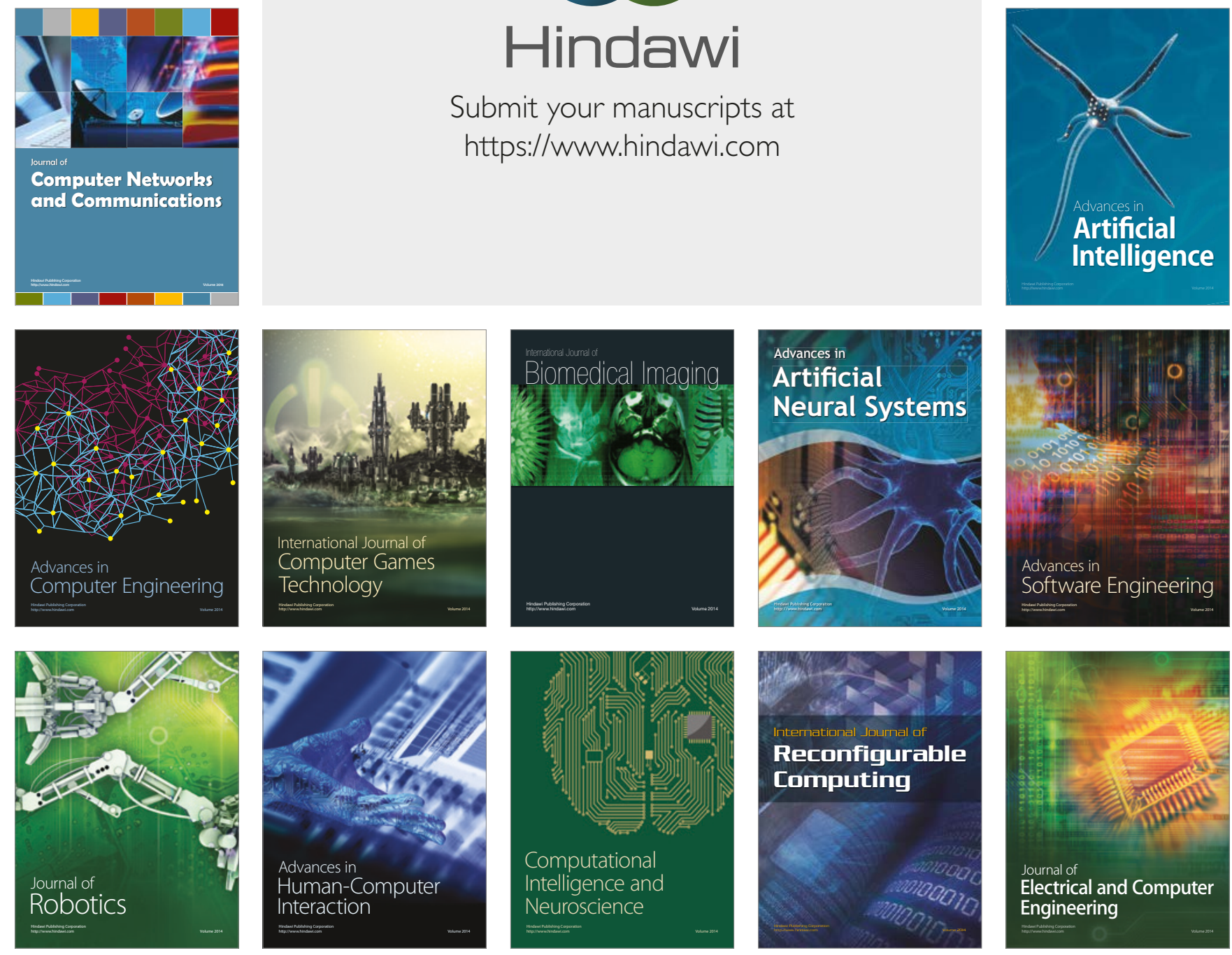